\title{
Proximal Versus Distal Ecological Stress: Socio-Ecological Influences on Political Freedom, Well-Being, and Societal Confidence in 159 Nations
}

\author{
Lucian Gideon Conway III $^{1}$, Linus Chan ${ }^{2}$, Shailee R. Woodard ${ }^{3}$, Mohsen Joshanloo ${ }^{4}$ \\ [1] Department of Psychology, University of Montana, Missoula, MT, USA. [2] Department of Psychology, University of Hong Kong, Hong Kong, China. \\ [3] Department of Psychology, Keene State College, Keene, NH, USA. [4] Department of Psychology, Keimyung University, Daegu, South Korea.
}

Journal of Social and Political Psychology, 2021, Vol. 9(1), 306-320, https://doi.org/10.5964/jspp.5927

Received: 2021-01-20 • Accepted: 2021-05-17 • Published (VoR): 2021-07-26

Handling Editor: Maria Fernandes-Jesus, University of Sussex, Falmer, United Kingdom

Corresponding Author: Lucian Gideon Conway III, 143 Skaggs, Department of Psychology, The University of Montana, Missoula, Montana 59812, USA. Email: luke.conway@umontana.edu

Supplementary Materials: Data, Materials [see Index of Supplementary Materials]

\begin{abstract}
Previous work from a socio-ecological perspective reveals that ecological stress has important effects on political, cultural, and psychological outcomes. However, that work has been limited by (1) a focus on distal forms of ecological stress that are hard for societies to control, and (2) a lack of large-scale conceptual replications. The present study aims to fill in these gaps by simultaneously testing the effects of both more distal ecological stress (e.g., climate) and more proximal ecological stress (e.g., water quality) on political restriction, political freedom, well-being, and societal confidence measurements. In a sample from the Gallup World Poll spanning over one and a half million participants and 159 nations, we found that while both kinds of ecological stress measurements predicted greater vertical political restriction, reduced horizontal political restriction, reduced well-being, and reduced freedom, only proximal forms of ecological stress predicted a loss in societal confidence. These results not only provide key conceptual replications of prior studies on new data, they also add previously unstudied outcomes and new ecological stressors. As a result, they help us better understand contributing factors to key societal issues such as freedom, well-being, and societal confidence.
\end{abstract}

\section{Keywords}

ecological stress, distal, proximal, freedom, well-being, confidence

Where do political and psychological differences across cultures come from? In the present paper, we evaluate ecological factors that might underlie differences across important issues at the intersection of social and political psychology: Freedom, well-being, and societal confidence. Better understanding the potential ecological origins of these key issues is a vital starting point for advancing our knowledge of social problems and their potential solutions.

While communication between persons undoubtedly influences the fate of cultural or political development (e.g., Bourgeois, 2002; Crandall, 1988; Harton \& Bourgeois, 2004; Kashima, 2000), a growing body of research suggests the roots of cultures also lie in farther-back ecological differences between regions. This socio-ecological perspective argues that because often people share natural and social ecologies on a large scale, these large-scale environments may exert cultural and political pressure on the individuals existing within that ecology - and so shape the direction a given political region takes (e.g., Oishi et al., 2017).

For example, work from a socio-ecological perspective suggests that individual freedom is restricted in cultures with both harsher climates (e.g., Van de Vliert \& Tol, 2014) and with more pathogen stress (e.g., Kusano \& Kemmelmeier, 2018; Schaller \& Murray, 2008). On the other hand, work with both harsh climates and frontier topography suggests that 
those ecological stressors sometimes produce more individualism and less governmental restriction (Conway et al., 2014; Kitayama et al., 2006; Kitayama et al., 2010; Van de Vliert, 2007, 2009, 2013).

This raises a puzzle: What factors might account for these potentially discrepant effects of various ecological stressors on cultural outcomes? Below, we discuss two types of processes to be evaluated in the present work: Those associated with an Ecological Dual-Pressure Model, and those associated with attributional blame processes.

\section{Ecological Dual-Pressure Model}

An Ecological Dual-Pressure Model (Chan \& Conway, 2018; Conway et al., 2017) suggests that all ecological stressors, despite having stressor-specific effects, each further have a general property that would concurrently pull culture in different directions. This model has its origins in work revealing that stress can produce multiple potential and seemingly competing responses. For example, research reveals that stress can sometimes produce close-minded ambiguity avoidance, yet at other times can produce open-minded attempts to solve problems (Gelfand et al., 2011; LePine et al., 2004; Richter \& Kruglanski, 2004; Van de Vliert, 2013). Other related work has validated dual-concern models in the conflict management and negotiation literatures (Blake \& Mouton, 1970; McClintock \& Moskowitz, 1976; Pruitt \& Rubin, 1986; Van de Vliert, 1997).

Building on this work, the Ecological Dual-Pressure model posits that ecological stress often produces predictable competing forces that operate concurrently in cultural development. For example, ecological stress both increases the need for interdependence (as we rely more on other people's help to overcome problems) and also increases the need for independence (as we strive to compete with others for scarce resources).

One possible implication of the Ecological Dual-Pressure Model was tested in a recent study (Conway et al., 2017). The model suggests that these concurrent competing processes could be captured in measurements related to governmental restriction. Specifically, some kinds of governmental restriction involve the degree that small groups of people impose their will on a populace (called vertical restriction), while other kinds of restrictions involve the degree that large groups of people vote to restrict themselves (called horizontal restriction). While in reality vertical/horizontal restriction is a contiuous variable, Conway et al. (2017) validated a group of horizontal restrictions that are more likely to apply in reality to large portions of the population (e.g., tax laws, speed limits) versus vertical restrictions that are likely to apply only to targeted minority groups (e.g., restrictions on LGBT marriage, laws targeting those convicted of crimes). The Ecological Dual-Pressure Model suggests that, regardless of stressor type, there should be a general tendency for ecological stressors to simultaneously pull vertical and horizontal restrictions in different directions increasing restrictions that benefit the majority group only (vertical) while simultaneously decreasing restrictions that might apply across group boundaries (horizontal).

To help better distinguish stressor-specific effects from effects that might be common across all stressors, this prior work (Conway et al., 2017) simultaneously compared the effects of climate stress, pathogen stress, and frontier topography stress on these two different types of political restriction measurements (both within the U.S. and across the world). While stressor-specific effects emerged consistent with prior theory, Conway et al. (2017) also found a general tendency for most stressors - captured in a summary ecological stress measure - to be positively predictive of vertical forms of political restriction, but negatively predictive of horizontal forms of political restriction. This result was consistent with the Ecological Dual-Pressure Model which argues that, no matter the kind of ecological stress, socio-ecological stressors simultaneously create dual pressures that pull vertical and horizontal dimensions in different directions (see Chan \& Conway, 2018; Conway et al., 2017, for discussions).

In the present study, we not only attempt replicate this finding using a new measurement of ecological stress (discussed below), we also extend the model to test ideas related to well-being. In particular, the model suggests that all ecological stressors are likely to make people feel less satisfaction with their lives - and yet it might make those same people more likely to seek other forms of well-being where they (for example) offer help to others in their local environment (described as Eudaimonic well-being; see Joshanloo, 2018b). In other words, we expect to see the dual-pressure tension in measurements that compare more direct measures of life satisfaction with well-being measurements evaluating other, less direct, ways that people find meaning. Ecological stress might decrease subjective life satisfaction while increasing other forms of meaning-making as people strive to cope with the stress. 


\section{An Attributional Perspective: The Importance of Controllability}

When considering the influence of socio-ecological stressors on cultural psychology, it is important to remember that most perceptions are filtered through a causal attributional lens (Eagly, Wood, \& Chaiken, 1978; Fein, 1996; Pryor, Rholes, Ruble, \& Kriss, 1984). Research suggests, for example, that people in the U.S. who attribute the $97 \%$ scientific consensus in favor of climate change to political motives are less likely to support environmentally-friendly legislation, a factor that predicts environmental policy preferences over and above political ideology (Conway \& Repke, 2019). Thus, it doesn't just matter what the surrounding social ecology is (in this case, what percentage of scientists endorse a point of view); it also matters what people are likely to attribute it to.

These reflections make it important to consider the match between ecological stressors and the potential that those stressors will be directly blamed - or not - on cultural factors (see also the related PAIR model specific to pandemics focusing on ideological matching; Conway et al., 2021). Indeed, some types of stressors may be inherently more likely to be chronically connected to attributional disounting for some kinds of beliefs. We focus here on one such set of potential stress-belief pairings: Controllable stressors and societal confidence.

Most of the prior research on ecological stress has focused on conceptually distal measurements of ecological stress. These measurements are considered distal because they are hypothesized to operate in a large historical context and are thus not easily proximally controlled by governments or populations (see, e.g., Conway et al., 2017; Van de Vliert \& Conway, 2019). Thus, the collective historical or scientific data that forms the backbone of most ecological stress measurements involves larger conceptual variables that presumably are not easily proximally influenced by the culture itself. Although of course everything can be potentially influenced by human behavior (e.g., global climate can be influenced by human activities), it is nonetheless the case that mountain ranges and climates are not easily altered by societal influences. As such, these distal measurements are less likely to be viewed by the populace as directly alterable by governments or societies, and thus we might expect less influence of these measurements on cultural judments related to confidence in one's society or government.

On the other hand, proximal stressors involve more easily-alterable processes that governments or society might reasonably be expected to have a larger sphere of control on (for discussion about water and air quality, see Keeler et al., 2012). Because of the high level of match between such measurements and potential attributional causes to societal confidence, it may be that they are particularly prone to influence such cultural judgments. For example, whereas governments cannot easily alter whether or not there is a hot or cold climate in general (distal), they can more easily alter the proximal water and air quality (see e.g., Keeler et al., 2012). Both involve socio-ecological stressors - both harsh climates and poor water/air quality provide stress that potentially impacts cultural development - and both potentially can be managed in some way by the government. But distal stressors are harder to directly control, whereas proximal stressors (while still of course not entirely in control of societies or governments) have a more reasonable match to attributions of blame, and in fact they are likely to be the targets of blame (see e.g., Keeler et al., 2012).

As a result, we expect that proximal measurements of stress would be viewed as more controllable and thus - only for cultural measurements such as societal confidence that are directly related to the potential attribution of blame we would expect those measurements to be more negatively impactful on societal confidence than distal measurements of ecological stress. This would suggest that attributional processes may moderate the causal line between top-down pressures and ultimate cultural change.

\section{The Present Study}

Prior studies (e.g., Oishi et al., 2017; Kitayama et al., 2006; Kitayama et al., 2010; Kusano \& Kemmelmeier, 2018; Schaller \& Murray, 2008; Van de Vliert, 2007, 2009, 2013) importantly reveal that natural ecologies influence the development of social and political systems, but they leave many gaps to fill. First, little prior work has evaluated the effects of more proximal ecological stress measurements, and research that has done so (e.g., Oishi et al., 2017) has not simultaneously evaluated proximal and distal ecological influences at the same time. To better understand the larger influence of ecological stress, it is important to have measurements that both report ecological stress at the distal level and that contain more proximal stressors that might more easily be altered by - or attributed to - governmental or populace 
action. This allows us to not only test the implications of the Ecological Dual-Pressure Model, but also the potential attributional moderators based on stressor-cultural belief match.

Even more broadly, there is generally a need for conceptual replications of existing theory and models. For example, Conway et al. (2017) tested their model using three types of ecological stressors and three political restriction measurements across nearly 200 nations and 50 U.S. states. While this covers quite a bit of ground, it is nonetheless still quite narrow, not only in that it does not have any clearly proximal measurements of stress, but also that it has a very narrow scope of dependent measurements of political and cultural variables. Thus, large-scale conceptual replications replications that take existing theory and concepts and test them with new variables on large datasets - are vital to the growth of accurate scientific knowledge (Crandall \& Sherman, 2016).

The present study helps fill in these gaps by using the Gallup World Poll (GWP), which allowed us to include over 1.5 million participants from 159 nations (Joshanloo 2018a, 2018b, 2019). In so doing, we pursue four research questions:

(1) Does proximal ecological stress show a similar pattern as distal ecological stress in predicting vertical and horizontal governmental restriction? First, in addition to using a summary measure of distal ecological stress (drawn from Conway et al., 2017), the present study uses a measurement of proximal ecological stress constructed from the GWP. This new measure of proximal ecological stress - which focuses on individual perceptions of air and water quality, an issue that people readily believe governments can directly affect (see e.g., Keeler et al., 2012) - allows for a conceptual replication test of Conway et al.'s basic effect of ecological stress on vertical versus horizontal forms of political restriction on a very different ecological stress measurement.

(2) Do proximal and distal ecological stress predict subjective measurements of freedom? Further, the present study also adds a new measurement of freedom that tests, not laws imbedded in each society, but rather individual perceptions of their own freedom. This allows for a novel test of the distal ecological stress measurement from Conway et al. (2017) on a conceptually-related - but methodologically very different - variable, as well as an independent test of our new proximal ecological stress measurement on this new freedom measurement.

(3) Do proximal and distal ecological stress predict direct and indirect measurements of well-being similarly? We further test the effects of both kinds of ecological stress on two types of health and well-being variables which allow for a more nuanced test of the Dual-Pressure Model.

(4) Do proximal and distal ecological stress differentially predict societal confidence? Finally, we test an additional variable on which it might be reasonable to expect, based on the attributional match approach discussed earlier, that the two types of ecological stress (distal versus proximal) would be differentially related: Confidence in social institutions. Specifically, to the degree that ecological stressors are perceived to be out of the control of societal or governmental institutions, we expect that increased ecological stress would have less of an impact on such confidence; but to the degree that ecological stressors are viewed as alterable by government, we might expect a strongly negative relationship between stress and societal confidence. Because our proximal forms of stress are, on average, more easily attributable to government or societal intervention (for a discussion of the overlap between water quality and societal intervention, see Keeler et al., 2012), this allows for an exploration of this idea.

\section{Gallup World Poll}

Our study utilizes the Gallup World Poll (GWP), which collects data from more than 159 countries annually. In these datasets, typically one thousand randomly sampled and nationally representative individuals over the age of 15 from each country are polled annually, and questions are translated using the back-translation technique. According to previous studies that used this dataset, the GWP samples represent more than $98 \%$ of the world's population (Diener \& Tay, 2015). This dataset has been used for prior research validated in a wide range of studies (e.g., Diener \& Tay, 2015; Helliwell et al., 2021; Joshanloo, 2018a, 2018b, 2019; Joshanloo \& Gebauer, 2019; Oishi \& Diener, 2014; Tay \& Diener, 2011), all of which analyzed data from dozens of countries to capture big trends.

Although no dataset can be fully equivalent across cultural contexts (e.g., Boehnke et al., 2014; Fischer \& Smith, 2021; Witte et al., 2020), the Gallup World Poll takes steps to ensure as much equivalence as reasonably possible, including measures to address equivalence, validity, and response bias (Tortora et al., 2010). Equivalence issues arise when research protocols differ based on language, data collection methods, or meaning of hypotheses (e.g. linguistic equivalence, procedural equivalence, theoretical equivalence). Validity issues arise when researchers try to measure the 
same constructs in different countries but face language issues or the lack of resources. Response bias issues arise when recruiting participants in some countries is easier than others, which often results in unequal sample sizes between countries.

Such problems are inevitable, but GWP data helps minimize these methodological issues in multiple ways. (1) First, data is collected with standardized procedures across different languages and nations where the questions are translated and back-translated (Tortora et al., 2010). In line with modern approaches to linguistic equivalence (Boehnke et al., 2014), this is not always a straight word-for-word translation, but rather takes the cultural context into account. (2) Further, GWP attempts to collect as respresentative of a sample as possible using standard methods for random selection of people within a country (Tortora et al., 2010). As Tortora and colleagues (2010, p. 539) said, "the GWP's driving design principle is to conduct nationally representative surveys in each country." While no such endeavor is perfect due to ever-shifting national conditions and sometimes-prohibitive costs, nonetheless GWP takes great pains each year, using accepted methods for random sampling, to collect as representative a sample as possible (see Tortora et al., 2010, for details). (3) Most of these interviews have historically been face-to-face around the world. Regardless of the type of interview, both face-to-face and telephone interviewers are comphrensively trained in strict protocols for selection and interviewing procedures. (4) Further, to ensure quality control, (a) supervisors accompany interviewers on a minimum of 2 interviews, (b) supervisors spot-check at least $5 \%$ of the cases by returning to the household in question and double-checking key information, (c) $25 \%$ of cases are back-checked by telephone or in person, and (d) $100 \%$ of questionnaire responses are checked for coherence and completeness.

Thus, while no data collection mechanism - and especially one covering so many nations - can possibly ensure perfect equivalence across samples and nations, GWP uses state-of-the-art, comprehensive methods to minimize the difficulties all researchers face when doing cross-cultural research. And indeed, recent empirical work suggests that, given these precautions, cross-national surveys can be reasonably used for cross-cultural comparisons (Welzel, Brunkert, Kruse, \& Inglehart, 2021).

It is for its rigor in minimizing cross-cultural research problems that the GWP in particular has been used extensively in cross-cultural comparison research, further validating its value (e.g., Diener \& Tay, 2015; Helliwell et al., 2021; Joshanloo, 2018a, 2018b, 2019; Joshanloo \& Gebauer, 2019; Oishi \& Diener, 2014; Tay \& Diener, 2011).

\section{Method}

The data analyzed in this study was collected by the Gallup World Poll from 2005 to 2017 in 159 countries. ${ }^{1}$ Gallup World Poll randomly surveys approximately 1,000 nationally representative residents from each participating country every year. Not all of these countries participated each year from 2005 to 2017, so there are slightly different amounts of data from year-to-year. The total sample for our study was comprised of over 1,500,000 individuals and 159 countries. In all cases, in line with prior research on the GWP (see, e.g., Helliwell et al., 2021), we use the nation as the unit of analyses by summarizing across multiple years within nation (see Helliwell et al., 2021). Summary scores for each nation were computed by first (a) averaging scores within-year for each nation, and then (b) averaging across years from 2005-2017. In this way, we produced a summary score for each variable for each nation.

Using the nation as the unit of analysis makes sense if the variables in question are not transient, day-to-day measurements (see Helliwell et al., 2021). For example, Helliwell and colleagues (2021) note that GWP well-being measurements (including those used here) are likely good candidates for aggregate nation-level measurements because they are based on more stable factors, whereas questions about transient emotional states are not. In the present research, we selected items that represent dimensions on which daily rapid change is unlikely (described in more detail below). Further, summarizing scores across multiple time periods increases the likelihood of capturing a stable cultural variable as opposed to something merely transient (Helliwell et al., 2021). ${ }^{2,3}$

1) The GWP data set is not public, and access has to be granted through Gallup. See: https://www.gallup.com/analytics/318875/global-research.aspx

2) While most items from the GWP are binary responses and thus questions of modality are not relevant, we computed relevant distribution statistics within each nation for two of the continuous variables from our dataset. As can be seen in the Supplementary Online Table (see Supplementary Materials), these 
With that in mind, dozens of the items from the GWP were selected for use a priori for the present study. The measures and individual items were aggregated in advance of performing analyses into four categories on the basis of conceptual overlap: (1) Proximal Ecological Stress, (2) Well-Being and Health, (3) Freedom, and (4) Confidence in Societal Institutions. These items were chosen because they matched the categories we were interested in. These aggregate measures and their items are described below.

\section{Independent Variables}

\section{Proximal Ecological Stressors (GWP)}

In two independent questions, participants were asked how much they were satisfied with their air and water quality, respectively. These two items were highly correlated and thus averaged into a single proximal ecological stress index, such that higher scores equated to less satisfaction with air and water quality (standardized $\alpha=.74){ }^{4}$

\section{Distal Ecological Stressors}

Distal ecological stress was measured via Conway et al.'s (2017) generalized ecological stress measurement. This measurement takes the average of five different ecological stressors: Extreme heat, extreme cold, pathogens, inland topography, and mountain topography (see Conway et al., 2017, for computational details and validation evidence).

\section{Dependent Variables}

\section{Political Restrictions}

The political restriction of each country was measured using Conway et al. (2017)'s indices for vertical and horizontal restriction. Vertical restriction was measured using two different indices. The first index compiled the country's laws surrounding abortion, LGBT rights, and the death penalty. These scores were then converted to $z$-scores and averaged to create a single vertical legal restriction index for each country. The higher the score for each of these three law categories, the more vertical restriction there is in the country (see Chan \& Conway, 2018; Conway et al., 2017). Additionally, a nation's level of vertical restriction was measured as in prior work (Chan \& Conway, 2018; Conway et al., 2017) by averaging the Freedom House political freedom indices for civil liberties and political rights as a continuous variable (higher scores $=$ more political restriction; Freedom House, 2013).

The horizontal restriction index is comprised of nation-wide laws on taxes, speed limits, and blood alcohol levels. These measures were converted to $z$-scores and averaged to produce an index of horizontal legal restriction for each nation (Conway et al., 2017; see also Chan \& Conway, 2018). Higher scores mean there is more horizontal restriction in the nation's laws.

\section{Freedom (GWP)}

Participants' psychological perception of cultural freedom was measured with four items from the GWP: sense of personal freedom to choose what one wishes to do with their life, whether or not the media has freedom or not, the degree to which people are afraid to openly express their political views (on a four-point scale from none to most; this item was reverse-scored), and whether or not the participant has voiced their opinion to a public official in the last

statistics suggest these variables are quite consistently normally distributed within each country in the GWP dataset. Further visual inspection of histograms additionally suggested these variables are uni-modal (and not multi-modal) in nature. In short, available data strongly suggests these data are useful to produce nation-level averages.

3) To illustrate the stability over time, we computed across-year correlations for all of our variables for 2015-2017. These correlations overwhelmingly illustrated the stability of these variables, with an average correlation of $r=.91$ and no variables at any point showing correlations below $r=.76$. In short, these variables are well-suited to summarize at the national level within-measure over time.

4) All standardized $\alpha$ s are computed at the nation-level. Because it is nation-level variance we are trying to understand, this is the appropriate unit for evaluating the across-nation consistency of the items. 
month. These were all converted to $z$-scores and averaged into a summary psychological freedom score (standardized $\alpha$ $=.78)$.

\section{Well-Being and Health (GWP)}

Participants' well-being and health was measured with a variety of scales and variables, including life satisfaction, personal safety, health problems, and satisfaction with income, health care, and other standards of life. In line with our prediction concerning differential effects of ecological stress on life satisfaction versus other kinds of meaning-making, we further subdivided these items into direct measurements of life satisfaction (including the primary "satisfaction ladder" measure used in the World Happiness Report, see Helliwell et al., 2021) and those related to Eudaimonic Well-Being. Direct measurements of life satisfaction (which were aggregated into a summary score for Cumulative Life Satisfaction) included items asking whether or not they are satisfied with healthcare, air and water quality, physical beauty, and standard of living. Eudaimonic well-being (Joshanloo, 2018b) was comprised of items involving learning ("Did you learn or do something interesting yesterday?"), social support ("If you were in trouble, do you have relatives or friends you can count on to help you whenever you need them, or not?"), respect ("Were you treated with respect all day yesterday?"), efficacy (“Can people in this country get ahead by working hard, or not?"), helping strangers (“In the past month, have you helped a stranger or someone you didn't know who needed help?"), and volunteering ("In the past month, have you volunteered your time to an organization?"). Participants were also asked additional items related to well-being, such as whether or not they felt safe walking alone at night where they live, whether or not they have health problems preventing them from "doing any of the things people [their] age normally can do," and how comfortably they feel living on their current household's income.

To compute an aggregate measure of all health and well-being items, all health and well-being items were converted to $z$-scores, reverse-scored (when necessary) so that higher scores always equaled greater health or well-being, and averaged into a summary well-being/health score (standardized $\alpha=.87){ }^{5}$

\section{Confidence in Societal Institutions (GWP)}

Lastly, confidence in societal institutions was measured by ten dichotomous items on the GWP. Participants were asked if they approved or disproved of the country leadership, if they were satisfied or dissatisfied with efforts to preserve the environment in their country, and whether or not they had confidence in the military, judiciary, government, financial institutions, and election honesty of their country. Participants were also asked whether or not corruption is widespread in their government and businesses and whether or not they felt the area they live in is a good place for people starting a new business. All items were converted to $z$-scores, reverse-scored (when necessary) so that higher scores always equaled greater confidence, and averaged into a summary societal confidence score (standardized $\alpha=.90$ ).

\section{Covariate: GDP}

To control for economic differences across nations, we further included a measurement of GDP/per capita. ${ }^{6}$

\section{Results}

Primary results for each kind of ecological stressor (distal versus proximal) are presented in Table 1.

5) Initially, one of the selected measures included the composite Eudaimonic well-being (EDW) measure created by Joshanloo (2018b). As seen below, one
of the EDW items more clearly fell conceptually into our "political freedom" category than our "well-being" category. To balance this potential tension, we
include this freedom-related item in our summary freedom category, but also independently report effects for the whole EDW scale. The summary health and
well-being scale, however, includes only the individual items from the EDW scale that fell into that category, and does not include the whole scale summary
(see Table 1, which identifies each item from the EDW scale for completeness).

6) We additionally controlled for the 2012 GINI index of income inequality. Unlike GDP, the GINI index was not strongly correlated with either distal ( $r=.17$ ) or proximal ( $r=.10$ ) ecological stress, and as a result, controlling for GINI did not meaningfully alter the pattern of results presented in Table 1 . In summary, controlling for GINI would not impact interpretation of our results in any way. These results largely appear to not be accounted for by income measurements. 
Table 1

Distal Versus Proximal Ecological Stressors Predicting DVs (Metric $=$ Pearson's $r$ )

\begin{tabular}{|c|c|c|c|c|}
\hline \multirow[b]{2}{*}{ Stressor Type } & \multicolumn{2}{|c|}{ Zero-Order } & \multicolumn{2}{|c|}{ Controlling for Wealth } \\
\hline & Distal & Proximal & Distal & Proximal \\
\hline \multicolumn{5}{|c|}{ Political Restriction (Conway et al., 2017) } \\
\hline Vertical Legal Restriction ${ }^{\mathrm{a}}$ & $.19^{*}$ & $.28^{* * *}$ & .05 & .10 \\
\hline Vertical Political Restriction $^{\mathrm{a}}$ & $.54^{* * *}$ & $.50^{* * *}$ & $.45^{* \star *}$ & $.35^{* * *}$ \\
\hline Horizontal Legal Restriction $^{\mathrm{a}}$ & $-.31^{* *}$ & $.12^{\dagger}$ & $-.24^{* * *}$ & -.01 \\
\hline \multicolumn{5}{|l|}{ Freedom (4 items) } \\
\hline Freedom $^{\mathrm{b}}$ & $-.27^{* * *}$ & $-.68^{* * *}$ & -.11 & $-.58^{* * *}$ \\
\hline Media Free & $-.36^{* * *}$ & $-.60^{* * *}$ & $-.19^{*}$ & $-.45^{* * *}$ \\
\hline Political Expression Allowed & $-.38^{* * *}$ & $-.44^{* * *}$ & $-.22^{* *}$ & $-.23^{* *}$ \\
\hline Voiced Opinion to Official & -.08 & $-.17^{*}$ & -.01 & -.08 \\
\hline CUMULATIVE FREEDOM & $-.35^{* * *}$ & $-.62^{* * *}$ & $-.18^{*}$ & $-.48^{* * *}$ \\
\hline \multicolumn{5}{|l|}{ Well-Being and Health (15 items) } \\
\hline Cumulative Life Satisfaction (5 its.) & $-.46^{* * *}$ & $-.69^{* * *}$ & $-.37^{* * *}$ & $-.32^{* * *}$ \\
\hline Satisfaction Ladder & $-.49^{* * *}$ & $-.54^{* * *}$ & $-.37^{* * *}$ & $-.32^{* * *}$ \\
\hline Satisfied Income & $-.40^{* * *}$ & $-.51^{* * *}$ & $-.21^{* *}$ & $-.28^{* * *}$ \\
\hline Satisfied Heal. Care & $-.38^{* * *}$ & $-.72^{* * *}$ & $-.21^{* *}$ & $-.62^{* * *}$ \\
\hline Satisfied Life & $-.31^{* * *}$ & $-.60^{* * *}$ & $-.12^{\dagger}$ & $-.44^{* * *}$ \\
\hline Satisfied with Physical Surround. & $-.43^{* * *}$ & $-.72^{* * *}$ & $-.28^{* * *}$ & $-.56^{* * *}$ \\
\hline Cumulative Eud. Well-Being (7 its.) & $-.27^{* * *}$ & $-.50^{* * *}$ & $-.14^{\dagger}$ & $-.38^{* * *}$ \\
\hline Felt Respect $^{\mathrm{b}}$ & $-.35^{* * *}$ & $-.24^{* *}$ & $-.23^{* *}$ & -.09 \\
\hline Learned Today ${ }^{\mathrm{b}}$ & $-.19^{*}$ & $-.30^{* * *}$ & -.11 & $-.21^{* *}$ \\
\hline Count on Help From Others ${ }^{b}$ & $-.37^{* *}$ & $-.38^{* * *}$ & $-.21^{* *}$ & $-.14^{\dagger}$ \\
\hline Gave Help to Others ${ }^{\mathrm{b}}$ & -.02 & -.02 & -.01 & -.01 \\
\hline Volunteered $^{\mathrm{b}}$ & $-.15^{\dagger}$ & $-.32^{* * *}$ & -.09 & $-.26^{* * *}$ \\
\hline Can Get Ahead With Hard Work ${ }^{\mathrm{b}}$ & .08 & $-.30^{* * *}$ & .07 & $-.37^{* * *}$ \\
\hline Safe at Night & $-.20^{*}$ & $-.40^{* * *}$ & -.06 & $-.25^{* *}$ \\
\hline Health Problems & $.20^{*}$ & $.21^{* *}$ & .07 & .02 \\
\hline CUMULATIVE WB\&H & $-.42^{* * *}$ & $-.67^{* * *}$ & $-.26^{* * *}$ & $-.55^{* * *}$ \\
\hline \multicolumn{5}{|c|}{ Confidence in Societal Institutions (10 items) } \\
\hline Satisfied Govt. Environmental & -.08 & $-.62^{* * *}$ & .01 & $-.58^{* * *}$ \\
\hline Confidence in Government & $.17^{*}$ & $-.28^{* * *}$ & $.16^{\dagger}$ & $-.37^{* * *}$ \\
\hline Confidence in Military & .00 & $-.32^{* * *}$ & .06 & $-.30^{* * *}$ \\
\hline Confidence in Financial & .12 & $-.28^{* * *}$ & .08 & $-.37^{* * *}$ \\
\hline Confidence in Judiciary & -.02 & $-.46^{* * *}$ & .10 & $-.39^{* * *}$ \\
\hline Confidence in Elections & $-.23^{* *}$ & $-.60^{* * *}$ & -.07 & $-.49^{* * *}$ \\
\hline Good Place for Business & $.14^{\dagger}$ & $-.23^{* *}$ & .11 & $-.31^{* * *}$ \\
\hline Approve Govt. Leadership & $.17^{* *}$ & $-.33^{* * *}$ & $.17^{*}$ & $-.39^{* * *}$ \\
\hline Government Corrupt & $.27^{* * *}$ & $.43^{* * *}$ & $.12^{\dagger}$ & $.26^{* * *}$ \\
\hline Business Corrupt & $.25^{* *}$ & $.41^{* * *}$ & .09 & $.24^{* *}$ \\
\hline CUMULATIVE CONF. S.I. & -.04 & $-.53^{* * *}$ & .06 & $-.48^{* * *}$ \\
\hline
\end{tabular}

Note. Distal = Distal ecological stressors (Conway et al., 2017); Proximal = Proximal ecological stressors (GWP).

${ }^{a}$ Distal (but not proximal) effects from identified row were also reported in Conway et al. (2017); they are reproduced here for comparative convenience, and all other findings reported in the table are entirely novel. ${ }^{\mathrm{b}}$ Item included in the Eud. Well-Being Scale. ${ }^{\dagger} p<.15 .{ }^{*} p \leq .05 .{ }^{* *} p \leq .01 .{ }^{* * *} p \leq .001$. 
As can be seen there, there is a great deal of overall similarity between the new Proximal Ecological Stress measurement (computed from the GWP) and the Distal Ecological Stress measurement from prior work (Conway et al., 2017). However, as outlined below, the attributional model that suggested a specific difference between the two kinds of measurements was also supported.

\section{Political Restriction}

For political restriction measurements, the Proximal Ecological Stress measurement showed a pattern consistent with past work. Specifically, Proximal Ecological Stress - like Distal Ecological Stress - showed positive and significant correlations with both measures of Vertical Restriction, but a negative correlation for Horizontal Restriction. Although the latter correlation approached (but did not obtain) statistical significance, Steiger's $Z$ tests confirmed that vertical and horizontal legal restriction showed the expected differential patterns for Proximal Ecological Stress: The correlation between Proximal Ecological Stress and both Vertical Legal Restriction and Vertical Horizontal Restriction was statistically significantly different than the correlation between Ecological Stress and Horizontal Legal Restriction $(z$ 's $>3.7, p$ 's $<.01$ ). This result conceptually replicates prior work on the Ecological Dual-Pressure Model (Conway et al., 2017) on a completely different ecological stress variable (air/water quality).

\section{Gallup World Poll Dependent Measures}

Results comparing both ecological stress measurements to measures of freedom, health, and institutional confidence also generally showed very similar patterns for the two types of ecological stress measurements, with the noted exception predicted by the attributional model.

Consistent with the results from the vertical restriction measurements, participants in countries with more ecological stressors (regardless of stressor type) felt less freedom (Proximal Ecological Stress correlation with Cumulative Freedom measure $r=-.62, p<.001 ; 95 \%$ CI [-.71, -.51]; Distal Ecological Stress correlation with Cumulative Freedom measure $r=-.35, p<.001 ; 95 \%$ CI $[-.48,-.21]$. This importantly suggests that not only do various kinds of ecological stress increase vertical political restriction (as indicated by restriction correlations from the prior section), but also that this lack of political freedom has psychological and behavioral consequences. It further suggests that vertical, but not horizontal, types of restriction are associated with the psychological reduction of freedom.

Consistent with discussion about the direct effects of ecological stress on individual health and well-being (e.g., Keeler et al., 2012), across multiple markers of health and well-being, ecological stressors (regardless of stressor type) were associated with poorer national health and well-being (Proximal Ecological Stress correlation with Cumulative Well-Being/Health measure $r=-.64, p<.001,95 \%$ CI [-.72, -.54]; Distal Ecological Stress correlation with Cumulative Well-Being/Health measure $r=-.42, p<.001,95 \%$ CI $[-.54,-.28]$.

The Ecological Dual-Pressure Model further predicted that both kinds of ecological stresss measurements would be differentially related to (1) direct measurements of life satisfaction (negative) and (2) more eudaimonic measurements related to well-being (positive). This hypothesis was only partially supported. Consistent with expectations, both kinds of ecological stress (proximal and distal) were strongly negatively related to direct measurements life satisfaction. Further, as Table 1 illustrates, also consistent with expectations, these relationships were not as negative for eudaimonic forms of well-being. However, the relationships were still significantly negative even for the total eudaimonic well-being scale. Thus, although the two were differentially related in the correct direction, there was no evidence of a reversal of the effect of stress on eudaimonic forms of well being as originally expected.

However, it is also worth noting that, considering the individual items on the eudaimonic scale, the farther one gets from individual measurements of satisfaction, the less negative the effects of stress get. For example, measurements such as those involving the individual respect people feel from others, or the help they feel like they can count on from others, show moderately negative relationships overall; but measurements about help given to others showed weaker negative relationships (and in three of four instances, those relationships were non-significant). Thus, these data partially conform to a more moderate view that ecological stressors do differentially impact the two types of well-being measurements in the expected direction, but the positive effect on the other kinds of meaning-making (such as helping others) is not strong enough to show a true reversal of the effect. 
As Table 1 reveals, consistent with the attributional model, the two types of ecological stressors had differential relationships with Confidence in Societal Institutions. Whereas Proximal Ecological Stress was associated with a loss of confidence in societal institutions (Proximal Ecological Stress correlation with the Cumulative Confidence in Societal Institutions measure $r=-.53, p<.001 ; 95 \%$ CI [-.63, -.41], Distal Ecological Stress was essentially uncorrelated with societal confidence measures (Distal Ecological Stress correlation with the Cumulative Confidence in Societal Institutions measure $r=-.04, p>.60 ; 95 \%$ CI $[-.19,+.12]$.

Notably, the largest difference in Table 1 between the two types of stressors occurred for the confidence in the government's environmental-based effort (for Proximal Stress, $r=-.62, p<.001,95 \%$ [-.71, -.51]; for Distal Stress, $r=-.08$, $p>.15,95 \% \mathrm{CI}[-.23,+.08]$. This is consistent with the attributional perspective discussed at the outset; this perspective suggests that people are more likely to blame government for air and water quality (our proximal measure) than they are for distal stressors (such as climate).

\section{Discussion}

Using a Gallup World Poll dataset that comprised 159 nations and over 1.5 million participants, the present study provided novel evidence of the importance of natural ecologies in understanding political and cultural differences across nations. In particular, it provided (a) triangulating evidence for the Ecological Dual-Pressure Model on vertical and horizontal restriction, (b) evidence for ecological impacts on health and well-being, and (c) initial evidence for potential differences in the effects of ecological stress on societal confidence for distal and proximal stress measurements. It provided weaker evidence for (d) the Ecological Dual-Pressure Model's prediction on differential impacts on types of well-being measurements. Below, we focus on the key issues in these results.

The results suggest a very similar pattern for both ecological stressor types on measurements related to political restriction and freedom. This overall similarity is itself important. Prior work (Conway et al., 2017) has suggested an Ecological Dual-Pressure Model for the influence of ecological stress on vertical and horizontal legal restriction. That model suggests that all ecological stressors, despite having stressor-specific effects, each further have a general property that would simultaneously increase vertical and decrease horizontal political restriction. The present study provides important additional evidence for this assertion by using more proximal (versus conceptually distal) measurements and completely different ecological stressors (water/air quality measurements). Indeed, given the conceptual and methodological distance from the original measurements of objective distal ecological stress, this similarity in pattern provides meaningful triangulating evidence for the Dual-Pressure Model's predictions regarding vertical and horizontal legal restriction.

Equally as importantly, these results have potential implications for the influence of ecological threats on culture moving forward. For example, consider the cultural and social psychology of the worldwide COVID-19 pandemic. Much research and theorizing has suggested that disease outbreaks influence cultural beliefs, with a largely unresolved debate centering around the potential causal influence of disease on ideological conservatism (e.g., Beall et al., 2016; Brenner \& Inbar, 2015; Helzer \& Pizarro, 2011; Martin, 2020; Oosterhoff et al., 2018; Shook et al., 2017; Tybur et al., 2016) and sometimes conflicting results for COVID-19 research in particular (e.g., Brownstein, 2020; Choma et al., 2021; Karwowski et al., 2020; Zettler et al., 2020).

Importantly, however, the present results suggest potential impacts of COVID-19 that may help us better understand the interface of COVID with culture that cuts across - and integrates - ideological lines. Specifically, these results suggest three possibilities moving forward. (1) First, the worldwide pandemic will reduce perceptions of political freedom. (2) Importantly, however, they further suggest - in line with the Dual-Pressure Model - that the lasting enacted governmental restrictions in response to COVID-19 will likely be more vertical than horizontal in nature. This latter hypothesis is interesting because it goes against what appears to largely be happening currently - and yet the Dual-Pressure Model suggests a different long-term outcome. (3) Finally, this present work suggests that both the reduction in perceptions of political freedom and the long-term increase in vertical (but not horizontal) restrictions will be greatest where COVID-19 hit the hardest. Each of these provides testable hypotheses moving forward that are drawn from the present work. 
While the wide difference between our Proximal and Distal Ecological Stress measurements makes the similarities across their effects in Table 1 more impressive, it also makes it harder to interpret their differences (an issue we return to in our limitations section below). Nevertheless, with that caution noted, the present results are at least consistent with an attributional match approach drawn from decades of attribution research (e.g., Conway \& Repke, 2019; Eagly, Wood, \& Chaiken, 1978; Fein, 1996; Pryor, Rholes, Ruble, \& Kriss, 1984). This approach suggests that different kinds of ecological stress may differentially affect societal confidence variables. This makes sense when one considers that conceptually proximal forms of ecological stress are likely viewed as more controllable by the government and society, and thus when those stressors are present, the ruling government is more likely to feel psychologically culpable to the people living under its rule. In contrast, the line between ecological stress and societal confidence is clunkier and less obvious for distal ecological stressors, and thus those stressors - while affecting many other aspects of political and cultural life - may have less of a role to play in governmental and societal confidence. As prior research has found in scenario studies (Conway \& Schaller, 2005; Conway \& Repke, 2019), this provides more real-world evidence that attributional processes may moderate the causal line between top-down pressures and ultimate cultural change.

\section{Limitations}

Like all studies, ours is not without limitations. We here discuss two. First, our proximal measure of ecological stress was not only more conceptually proximal (as outlined earlier, this means that it is more likely controllable in the short-term), but also was more subjective in nature. Our distal ecological stress measures were comprised of objective data, whereas our proximal measure was comprised of subjective reports about stressors. This is a strength in many regards as most prior studies have used methodologically objective measurements of ecological variables (for discussions on the importance of the distinction between objective and subjective measurements of culture-relevant variables, see Delle Fave, 2016; Guerini \& Nuvolati, 2016; for a counter-example, see Tybur et al., 2016) and thus more subjective measurements are needed. Indeed, objective measurements are at best proxies of the proposed psychological mechanisms, and as such are necessarily psychologically imprecise (see Van de Vliert \& Conway, 2019).

In spite of these strengths, however, it does pose an intepretational problem when interpreting differences in effects across the two types of measures. We use measurements here that are both subjective and proximal (compared to measurements that are both objective and distal). Thus, methodological subjectivity and conceptual proximity are essentially perfectly overlapping in the present study, and thus it is hard to tease the two apart. While the available evidence suggests that conceptual proximity might play an important role, ${ }^{7}$ the present study cannot definitively tease apart these explanations.

Another limitation is that the data are correlational in nature, and thus we must be cautious in drawing causal conclusions. Although we are testing a socio-ecological perspective that assumes a causal effect of ecological environments on political and cultural systems (see, e.g., Van de Vliert \& Conway, 2019) - and our data are consistent with that causal path - we (as with most work in the field) cannot nonetheless draw firm causal conclusions.

Equally as importantly, our proximal and distal ecological stress measures might be in a different position with respect to the causal issue. Specifically, whereas it is harder to imagine a reverse causal path for distal measures like climate (whereby, say, perceptions of freedom influence climate), it is easier to imagine a causal path for our proximal measurements (whereby, say, perceptions of freedom influences perceptions of air quality). While we cannot of course confidently rule out the reverse causal explanation in those cases - and thus caution is warranted - the overall pattern of results tested here is consistent with a view that moves from ecological stress to our outcome measures. Indeed, triangulation plays a role here - the overall similarity in the pattern of the distal measures (where reverse causality is not probable) and our new proximal measure provides a larger picture suggesting an ecological stress $\rightarrow$ outcome path is the most likely. It is worth remembering in this context that the methodological distance for tests of the Dual-Pressure Model between ecological stress and political restriction is actually greater (in that case) for proximal ecological stress measurements than distal ones. Yet, in spite of that, distal ecological stress showed the same pattern. Thus, although

7) In an online supplement, we used an objective measurement of air/water quality to try and tease these apart. This provided mixed evidence (see Supplementary Materials). 
reverse causality cannot be ruled out, the overall pattern emerging here suggests ecological stress as the most likely causal factor.

\section{Concluding Thoughts}

Socio-ecological perspectives can help us understand where cultural and political differences come from. Yet research on these perspectives sometimes lacks large-scale comparative studies that use multiple kinds of methods to test conceptually related but methodologically distinct variables. The present study helps fill in this gap by combining a highly-validated, large-scale dataset with previously-generated data used by socio-ecological researchers. The results suggest continued support for one of the main predictions of the Ecological Dual-Pressure Model - that there is a general property of ecological stress which concurrently pulls vertical and horizontal legal restriction in different directions - while further revealing potential attributional differences in effects for proximal versus distal forms of ecological stress.

Funding: This work was supported by the Ministry of Education of the Republic of Korea and the National Research Foundation of Korea (NRF-2017S1A3A2066611).

Acknowledgments: The authors have no additional (i.e., non-financial) support to report.

Competing Interests: The authors have declared that no competing interests exist.

Data Availability: For this article, a dataset is freely available (Conway, Chan, Woodard, \& Joshanloo, 2021b).

\section{Supplementary Materials}

The Supplementary Materials comprise two documents (for access see Index of Supplementary Materials below):

1. Additional analyses separating out the proximal/distal dimension from the subjective/objective dimension. Specifically, we present analyses and interpretation of a water/air measurement that is proximal-but-objective.

2. A supplementary data file containing skewness and kurtosis data within each country in our sample for the two measurements for which such measurements are possible. Specifically, we present within-country skewness and kurtosis for eudaimonic wellbeing ("eud" on the datafile) and for the satisfaction ladder ("ladder" on the datafile).

\section{Index of Supplementary Materials}

Conway, L. G., Chan, L., Woodard, S. R., \& Joshanloo, M. (2021a). Supplementary materials to "Proximal versus distal ecological stress: Socio-ecological influences on political freedom, well-being, and societal confidence in 159 nations" [Materials]. PsychOpen GOLD. https://doi.org/10.23668/psycharchives.4966

Conway, L. G., Chan, L., Woodard, S. R., \& Joshanloo, M. (2021b). Supplementary materials to "Proximal versus distal ecological stress: Socio-ecological influences on political freedom, well-being, and societal confidence in 159 nations" [Research data]. PsychOpen GOLD. https://doi.org/10.23668/psycharchives.4967 


\section{References}

Beall, A. T., Hofer, M. K., \& Schaller, M. (2016). Did the Ebola outbreak influence the 2014 U.S. Federal Elections (and if so, how)? Psychological Science, 27, 595-605. https://doi.org/10.1177/0956797616628861

Blake, R. R., \& Mouton, J. S. (1970). The fifth achievement. The fournal of Applied Behavioral Science, 6(4), 413-426. https://doi.org/10.1177/002188637000600403

Boehnke, K., Arnaut, C., Bremer, T., Chinyemba, R., Kiewitt, Y., Koudadjey, A. K., . . Neubert, L. (2014). Toward emically informed cross-cultural comparisons: A suggestion. Journal of Cross-Cultural Psychology, 45(10), 1655-1670.

https://doi.org/10.1177/0022022114547571

Bourgeois, M. (2002). Heritability of attitudes constrains dynamic social impact. Personality and Social Psychology Bulletin, 28, 1063-1072. https://doi.org/10.1177/01461672022811005

Brenner, C. J., \& Inbar, Y. (2015). Disgust sensitivity predictspolitical ideology and policy attitudes in The Netherlands. European fournal of Social Psychology, 45, 27-38. https://doi.org/10.1002/ejsp.2072

Brownstein, R. (2020, March 20). Red and blue America aren't experiencing the same pandemic. The Atlantic. Retrieved from https://www.theatlantic.com/politics/archive/2020/03/how-republicans-and-democrats-think-about-coronavirus/608395

Chan, L., \& Conway, L. G., III. (2018). Autocratic government moderates the relationship between culture and legal restriction. fournal of Cross-Cultural Psychology, 49(9), 1457-1463. https://doi.org/10.1177/0022022118793538

Choma, B. L., Hodson, G., Sumantry, D., Hanoch, Y., \& Gummerum, M. (2021). Ideological and psychological predictors of COVID-19related collective action, opinions, and health compliance across three nations. fournal of Social and Political Psychology, 9(1), 123-143. https://doi.org/10.5964/jspp.5585

Conway, L. G., III, Bongard, K., Plaut, V., Gornick, L. J., Dodds, D. P., Giresi, T., . . Houck, S. C. (2017). Ecological origins of freedom: Pathogens, heat stress, and frontier topography predict more vertical but less horizontal governmental restriction. Personality and Social Psychology Bulletin, 43, 1378-1398. https://doi.org/10.1177/0146167217713192

Conway, L. G., III, Houck, S. C., \& Gornick, L. J. (2014). Regional differences in individualism and why they matter. In J. Rentfrow (Ed.), Psychological geography (pp. 31-50). Washington, DC, USA: American Psychological Association.

Conway, L. G., III, \& Repke, M. A. (2019). The psychological contamination of pro-environmental consensus: Political pressure for environmental belief agreement undermines its long-term power. fournal of Environmental Psychology, 62, 12-21. https://doi.org/10.1016/j.jenvp.2019.02.001

Conway, L. G., III, \& Schaller, M. (2005). When authorities' commands backfire: Attributions about consensus and effects on deviant decision making. Journal of Personality and Social Psychology, 89(3), 311-326. https://doi.org/10.1037/0022-3514.89.3.311

Conway, L. G., III, Woodard, S. R., Zubrod, A., \& Chan, L. S. (2021). Why are conservatives less concerned about the Coronavirus (COVID-19) than liberals? Comparing political, experiential, and partisan messaging explanations. Personality and Individual Differences. Advance online publication. https://doi.org/10.1016/j.paid.2021.111124

Crandall, C. S. (1988). Social contagion of binge eating. fournal of Personality and Social Psychology, 55, 588-598. https://doi.org/10.1037/0022-3514.55.4.588

Crandall, C. S., \& Sherman, J. W. (2016). On the scientific superiority of conceptual replications for scientific progress. fournal of Experimental Social Psychology, 66, 93-99. https://doi.org/10.1016/j.jesp.2015.10.002

Delle Fave, A. (2016). Subjective indicators of well-being: Conceptual background and applications in social sciences. In L. Bruni \& P. L. Porta (Ed.), Handbook of research methods and applications in happiness and quality of life (pp. 71-89). Cheltenham, United Kingdom: Edward Elgar.

Diener, E., \& Tay, L. (2015). Subjective well-being and human welfare around the world as reflected in the Gallup World Poll. International fournal of Psychology, 50(2), 135-149. https://doi.org/10.1002/ijop.12136

Eagly, A. H., Wood, W., \& Chaiken, S. (1978). Causal inferences about communicators and their effect on opinion change. fournal of Personality and Social Psychology, 36(4), 424-435. https://doi.org/10.1037/0022-3514.36.4.424

Fein, S. (1996). Effects of suspicion on attributional thinking and the correspondence bias. fournal of Personality and Social Psychology, 70, 1164-1184. https://doi.org/10.1037/0022-3514.70.6.1164

Fischer, R., \& Smith, P. B. (2021). How far can measurement be culture-free? In M. Bender \& B. G. Adams (Eds.), Methods and assessment in culture and psychology (pp. 319-340). https://doi.org/10.1017/9781108675475.016

Freedom House. (2013). Freedom in the world country ratings. Retrieved from http://www.freedomhouse.org 
Gelfand, M. J., Raver, J. L., Nishii, L., Leslie, L. M., Lun, J., Lim, B. C., . . Yamaguchi, S. (2011). Differences between tight and loose cultures: A 33-nation study. Science, 332, 1100-1104. https://doi.org/10.1126/science.1197754

Guerini, M., \& Nuvolati, G. (2016). Happiness, subjective and objective indicators. In L. Bruni \& P. L. Porta (Eds.), Handbook of research methods and applications in happiness and quality of life (pp. 400-418). Cheltenham, United Kingdom: Edward Elgar.

Harton, H. C., \& Bourgeois, M. J. (2004). Cultural elements emerge from dynamic social impact. In M. Schaller \& C. S. Crandall (Eds.), The psychological foundations of culture (pp. 41-76). Mahwah, NJ, USA: Lawrence Erlbaum Associates.

Helliwell, J. F., Huang, H., Wang, S., \& Norton, M. (2021). World happiness, trust and deaths under COVID-19. In World Happiness Report (pp. 13-56). New York, NY, USA: Sustainable Development Solutions Network. Retrieved from https://worldhappiness.report/ed/2021/happiness-trust-and-deaths-under-covid-19

Helzer, E. G., \& Pizarro, D. A. (2011). Dirty liberals! Reminders of physical cleanliness influence moral and political attitudes. Psychological Science, 22, 517-522. https://doi.org/10.1177/0956797611402514

Joshanloo, M. (2018a). Income satisfaction is less predictive of life satisfaction in individuals who believe their lives have meaning or purpose: A 94-nation study. Personality and Individual Differences, 129, 92-94. https://doi.org/10.1016/j.paid.2018.03.018

Joshanloo, M. (2018b). Optimal human functioning around the world: A new index of eudaimonic well-being in 166 nations. British Journal of Psychology, 109, 637-655. https://doi.org/10.1111/bjop.12316

Joshanloo, M. (2019). Cultural religiosity as the moderator of the relationship between affective experience and life satisfaction: A study in 147 countries. Emotion, 19(4), 629-636. https://doi.org/10.1037/emo0000469

Joshanloo, M., \& Gebauer, J. E. (2019). Religiosity’s nomological network and temporal change: Introducing an extensive country-level religiosity index based on Gallup World Poll data. European Psychologist, 25(1), 26-40. https://doi.org/10.1027/1016-9040/a000382

Karwowski, M., Kowal, M., Groyecka, A., Białek, M., Lebuda, I., Sorokowska, A., \& Sorokowski, P. (2020). When in danger, turn right: COVID-19 threat promotes social conservatism and right-wing presidential candidates. PsyArXiv. https://doi.org/10.31234/osf.io/pjfhs

Kashima, Y. (2000). Maintaining cultural stereotypes in the serial reproductions of narratives. Personality and Social Psychology Bulletin, 26, 594-604. https://doi.org/10.1177/0146167200267007

Keeler, B. L., Polasky, S., Brauman, K. A., Johnson, K. A., Finlay, J. C., O’Neill, A., . . Dalzell, B. (2012). Linking water quality and wellbeing for improved assessment and valuation of ecosystem services. Proceedings of the National Academy of Sciences of the United States of America, 109(45), 18619-18624. https://doi.org/10.1073/pnas.1215991109

Kitayama, S., Conway, L. G., III, Pietromonaco, P. R., Park, H., \& Plaut, V. C. (2010). Ethos of independence across regions in the United States: The production-adoption model of cultural change. The American Psychologist, 65, 559-574. https://doi.org/10.1037/a0020277

Kitayama, S., Ishii, K., Imada, T., Takemura, K., \& Ramaswamy, J. (2006). Voluntary settlement and the spirit of independence: Evidence from Japan's “Northern frontier.” Journal of Personality and Social Psychology, 91, 369-384. https://doi.org/10.1037/0022-3514.91.3.369

Kusano, K., \& Kemmelmeier, M. (2018). Ecology of freedom: Competitive tests of the role of pathogens, climate, and natural disasters in the development of socio-political freedom. Frontiers in Psychology, 9, Article 954. https://doi.org/10.3389/fpsyg.2018.00954

LePine, J. A., LePine, M. A., \& Jackson, C. L. (2004). Challenge and hindrance stress: Relationships with exhaustion, motivation to learn, and learning performance. The fournal of Applied Psychology, 89, 883-891. https://doi.org/10.1037/0021-9010.89.5.883

Martin, C. C. (2020). How education did (and did not) accentuate partisan differences during the Ebola outbreak of 2014-15. Journal of Social and Political Psychology, 8, 108-131. https://doi.org/10.5964/jspp.v8i1.1072

McClintock, C. G., \& Moskowitz, J. M. (1976). Children's preferences for individualistic, cooperative, and competitive outcomes. Journal of Personality and Social Psychology, 34(4), 543-555. https://doi.org/10.1037/0022-3514.34.4.543

Oishi, S., \& Diener, E. (2014). Residents of poor nations have a greater sense of meaning in life than residents of wealthy nations. Psychological Science, 25(2), 422-430. https://doi.org/10.1177/0956797613507286

Oishi, S., Yagi, A., Komiya, A., Kohlbacher, F., Kusumi, T., \& Ishii, K. (2017). Does a major earthquake change job preferences and human values? European fournal of Personality, 31, 258-265. https://doi.org/10.1002/per.2102

Oosterhoff, B., Shook, N. J., \& Ford, C. (2018). Is that disgust I see? Political ideology and biased visual attention. Behavioural Brain Research, 336, 227-235. https://doi.org/10.1016/j.bbr.2017.09.005

Pruitt, D. G., \& Rubin, J. Z. (1986). Social conflict: Escalation, stalemate, and settlement. New York, NY, USA: Random House.

Pryor, J. B., Rholes, W. S., Ruble, D. N., \& Kriss, M. (1984). A developmental analysis of salience and discounting in social attribution. Representative Research in Social Psychology, 14, 30-40. 
Richter, L., \& Kruglanski, A. W. (2004) Motivated closed mindedness and the emergence of culture. In M. Schaller \& C. S. Crandall (Eds.), The psychological foundations of culture (pp. 101-121). Mahwah, NJ, USA: Lawrence Erlbaum Associates.

Schaller, M., \& Murray, D. R. (2008). Pathogens, personality and culture: Disease prevalence predicts worldwide variability in sociosexuality, extraversion, and openness to experience. fournal of Personality and Social Psychology, 95, 212-221. https://doi.org/10.1037/0022-3514.95.1.212

Shook, N. J., Oosterhoff, B., Terrizzi, J. A., Jr., \& Brady, K. M. (2017). "Dirty politics”: The role of disgust sensitivity in voting. Translational Issues in Psychological Science, 3(3), 284-297. https://doi.org/10.1037/tps0000111

Tay, L., \& Diener, E. (2011). Needs and subjective well-being around the world. Fournal of Personality and Social Psychology, 101(2), 354-365. https://doi.org/10.1037/a0023779

Tortora, R., Srinivasan, R., \& Esipova, N. (2010). The Gallup World Poll. In J. Harkness, M. Braun, \& B. Edwards (Eds.), Survey methods in multinational, multiregional contexts (pp. 535-544). Hoboken, NJ, USA: John Wiley \& Sons.

Tybur, J. M., Inbar, Y., Aarøe, L., Barclay, P., Barlow, F. K., De Barra, M., . . Consedine, N. S. (2016). Parasite stress and pathogen avoidance relate to distinct dimensions of political ideology across 30 nations. Proceedings of the National Academy of Sciences of the United States of America, 113, 12408-12413. https://doi.org/10.1073/pnas.1607398113

Van de Vliert, E. (1997). Complex interpersonal conflict behaviour: Theoretical frontiers. Hove, United Kingdom: Psychology Press.

Van de Vliert, E. (2007). Climatoeconomic roots of survival versus self-expression cultures. fournal of Cross-Cultural Psychology, 38, 156-172. https://doi.org/10.1177/0022022106297298

Van de Vliert, E. (2009). Climate, affluence, and culture. New York, NY, USA: Cambridge University Press.

Van de Vliert, E. (2013). Climato-economic habitats support patterns of human needs, stresses, and freedoms. Behavioral and Brain Sciences, 36, 465-480. https://doi.org/10.1017/S0140525X12002828

Van de Vliert, E., \& Conway, L. G., III. (2019). Northerners and Southerners differ in conflict culture. Negotiation and Conflict Management Research, 12(3), 256-277. https://doi.org/10.1111/ncmr.12138

Van de Vliert, E., \& Tol, R. S. J. (2014). Harsh climate promotes harsh governance (except in cold-dry-wealthy environments). Climate Research, 61, 19-28. https://doi.org/10.3354/cr01246

Welzel, C. W., Brunkert, L., Kruse, S., \& Inglehart, R. F. (2021). Non-invariance? An overstated problem with misconceived causes. Sociological Methods \& Research. Advance online publication. https://doi.org/10.1177/0049124121995521

Witte, E. H., Adrian, S., \& Boehnke, K. (2020). A new empirical approach to intercultural comparisons of value preferences based on Schwartz's theory. Frontiers in Psychology, 11, Article 1723. https://doi.org/10.3389/fpsyg.2020.01723

Zettler, I., Schild, C., Lilleholt, L., \& Böhm, R. (2020). Individual differences in accepting personal restrictions to fight the COVID-19 pandemic: Results from a Danish adult sample. PsyArXiv. https://doi.org/10.31234/osf.io/pkm2a 